\section{Heterogenes Outcome nach Sklerotherapie}

Khaitovich B, Kalderon E, Komisar O et al. Venous Malformations Sclerotherapy: Outcomes, Patient Satisfaction and Predictors of Treatment Success. Cardiovasc Intervent Radiol 2019; 42 (12): 16951701. doi:10.1007/s00270-019-02338-y

Venöse Malformationen sind der häufigste Subtyp von Gefäßmalformationen. Obwohl sie angeboren sind, können sie sich in jedem Alter manifestieren, mit einer hohen Heterogenität hinsichtlich Lokalisation und Größe. Außerdem haben sie möglicherweise einen wesentlichen Einfluss auf die Lebensqualität. Die perkutane Sklerotherapie ist aktuell die Methode der Wahl zur Behandlung venöser Malformationen.

Autoren um Khaitowhich untersuchten in ihrer retrospektiven Studie die Ergebnisse der Sklerotherapie bei Patienten mit venösen Malformationen hinsichtlich Patientenzufriedenheit, Komplikationen und Prädiktoren für ein positives Ansprechen auf die Therapie. Sie analysierten dafür 309 Patienten in ihrem Zentrum zwischen
2002 und 2014 mittels eines Fragebogens zur Selbstevaluation.

\section{Ergebnisse}

Insgesamt antworteten 153 Patienten (mittleres Alter 21 Jahre; 6 Wochen bis 67 Jahre). 64 Patienten (42\%) hatten eine venöse Malformation in der unteren Extremität, 35 (23\%) im Kopf-Hals-Bereich, 33 Patienten (22\%) am Stamm, 14 (9\%) an der oberen Extremität und 5 (3\%) im Bereich des Gesäßes oder der Genitalien. Eine komplette Linderung der Schwellung, der Schmerzen, der funktionellen und ästhetischen Beschwerden wurde bei $23 \%$, $22 \%, 17 \%$ bzw. $12 \%$ berichtet. 38 Patienten ( $25 \%$ ) berichteten über zufriedenstellende Behandlungsoutcomes, 55 (36\%) waren zufrieden, 49 (32\%) waren nicht zufrieden, 11 (7\%) waren sehr unzufrieden. Signifikante Korrelationen zwischen Patientenzufriedenheit und demografischen Charakteristiken, Läsionslokalisation und -größe, Gewebebeteiligung oder der Menge des verwendeten Sklerosans konnten nicht gefunden werden. Die Rate der Grad-III-Komplikationen war 7\%, der Grad-IV-Komplikationen $2 \%$.

\section{FAZIT}

Die Autoren schließen, dass die Sklerotherapie die Behandlungsoption der ersten Wahl für die meisten Patienten mit venöser Malformation ist. Allerdings ergab sich in ihrer Arbeit eine erwähnenswerte Versagensrate aus Patientensicht. Die Sklerotherapie verbessert zumindest einige Symptome bei der Mehrheit der Patienten. Allerdings sind zeitlich begrenzte und dauerhafte Komplikationen häufig. In Übereinstimmung mit der bestehenden Literatur ist es nach wie vor schwer vorherzusagen, welche Läsionen oder Patienten besser auf die Sklerotherapie ansprechen. Daher sind, wie die Autoren ergänzen, realistische Erwartungen auf Behandler- und Patientenseite entscheidend für die Patientenzufriedenheit.

MOR Dr. med. Benedikt Lampl, Regensburg 